ISSN 0103-9954

\title{
REMOÇÃO DE HEMICELULOSES DA MADEIRA POR TRATAMENTO DE AUTO-HIDRÓLISE
}

\author{
WOOD CELLULOSE REMOVAL BY MEANS OF SELF- HYDROLYSIS TREATMENT
}

Dalton Longue Júnior ${ }^{1}$ Jorge Luiz Colodette ${ }^{2}$

\begin{abstract}
RESUMO
O objetivo deste estudo foi avaliar o impacto da remoção de hemiceluloses de cavacos de madeira de um híbrido de duas espécies de eucalipto (Eucalyptus grandis $x$ Eucalyptus urophylla), por tratamento de autohidrólise $\left(\mathrm{H}_{2} \mathrm{O}\right)$ dos mesmos, no comportamento do processo kraft subsequente e na branqueabilidade e qualidade das polpas. Os tratamentos de auto-hidrólise dos cavacos foram realizados em temperaturas de $152^{\circ} \mathrm{C}\left(30,45\right.$ e 60 minutos); $160^{\circ} \mathrm{C}\left(15,30\right.$ e 45 minutos); e $170^{\circ} \mathrm{C}(5,10,15,20$ e 30 minutos); relação água/madeira de $4: 1 \mathrm{~m}^{3} / \mathrm{t}$. Cavacos normais (referência) e auto-hidrolisados a $170^{\circ} \mathrm{C}$ por 5,15 e $30 \mathrm{~min}$ foram cozidos pelo processo kraft até número kappa 16 - 18 e a polpa resultante foi branqueada pela sequência $\mathrm{O} / \mathrm{OD}(\mathrm{EPO}) \mathrm{DD}$. Os resultados indicaram que a auto-hidrólise dos cavacos por 30 minutos a $170{ }^{\circ} \mathrm{C}$, permitiu a remoção de até $60 \%$ de hemiceluloses. O rendimento dos cozimentos de cavacos autohidrolisados por 30 minutos foi cerca de $6 \%$ menor e o teor de pentosanas $88 \%$ menor que o dos cavacos normais. A eficiência da deslignificação com oxigênio da polpa derivada de cavacos auto-hidrolisados por 30 minutos foi de $75 \%$, contra $43,6 \%$ da polpa-referência, e o custo de branqueamento pela sequência $\mathrm{O} /$ OD(EPO)DD foi US\$ 7/tas de polpa mais baixo que o da polpa-referência. O efluente do branqueamento da polpa oriunda de cavacos auto-hidrolisados apresentou menores valores de DQO $(39,6 \%)$, cor $(21,3 \%)$ e $\operatorname{AOX}(51,6 \%)$, em relação ao da polpa-referência.
\end{abstract}

Palavras-chave: auto-hidrólise; remoção de hemiceluloses; xilanas; rendimento.

\begin{abstract}
The objective of this study was to assess the impact of removing hemicelluloses from chips of eucalyptus wood by self-hydrolysis treatment $\left(\mathrm{H}_{2} \mathrm{O}\right)$ and on the subsequent ' $\mathrm{kraft}$ ' process behavior and pulps bleachability and quality. The self-hydrolysis treatments were conducted at temperatures of $152^{\circ} \mathrm{C}(30,45$ and 60 minutes); $160^{\circ} \mathrm{C}\left(15,30\right.$ and 45 minutes); and $170^{\circ} \mathrm{C}(5,10,15,20$ and 30 minutes); water: wood ratio of $4: 1 \mathrm{~m}^{3} / \mathrm{t}$. Normal chips (reference) and self-hydrolyzed chips at $170^{\circ} \mathrm{C}$ during 5,15 and 30 minutes were submitted to 'kraft' cooking up to kappa number 16 - 18 and the resulting pulp was bleached using the O/OD (EPO) DD sequence. According to the results obtained, self-hydrolyzing the chips at $170^{\circ} \mathrm{C}$ for 30 minutes allowed the removal of up to $60 \%$ hemicelluloses. Cooking yield of the self-hydrolyzed chips for 30 minutes was around $6 \%$ smaller and pentosan content $88 \%$ lower than that of the normal chips. The efficiency of delignification with oxygen of the pulp derived from self-hydrolyzed chips for 30 minutes was of $75 \%$, compared to $43.6 \%$ of the reference-pulp, and the bleaching cost using the O/OD (EPO) DD sequence was US\$7/adt per pulp, lower that that of the reference-pulp. The effluent originated from bleaching the pulp derived from the self-hydrolyzed chips presented lower values of COD (39.6\%), color $(21.3 \%)$ and AOX (51.6\%), compared to that of the reference-pulp.
\end{abstract}

Keywords: self-hydrolysis; hemicellulose removal; xylans; cooking yield.

1. Engenheiro Florestal, M.Sc., Doutorando em Ciência Florestal, Laboratório de Celulose e Papel, Universidade Federal de Viçosa, Av. P.H. Rolfs, s/n, Campus, CEP 36570-000, Viçosa (MG). daltonufv@yahoo.com.br

2. Engenheiro Florestal, Ph.D., Professor Titular do Departamento de Engenharia Florestal, Laboratório de Celulose e Papel, Universidade Federal de Viçosa, Av. P.H. Rolfs, s/n, Campus, CEP 36570-000, Viçosa (MG). colodett@ufv.br

Recebido para publicação em 25/05/2009 e aceito em 30/08/2010 


\section{INTRODUÇÃO}

A eficiência econômica de utilização da madeira de eucalipto para produção de celulose kraft pode ser melhorada, aplicando-se o conceito da biorrefinaria. O conceito baseia-se na utilização de componentes da madeira que são subutilizados durante a produção de celulose para fabricar produtos nobres de alto valor agregado, como alcoóis, xilitol, biopolímeros, produtos químicos básicos etc., mediante a extração e o processamento das hemiceluloses da madeira (ROOKS, 2004).

Para madeiras de eucalipto, a perda de hemiceluloses durante a polpação kraft pode alcançar valores consideráveis (50-60\%). A perda significativa de hemiceluloses durante a polpação se deve a vários fatores inerentes à sua estrutura química, como baixo peso molecular, baixa cristalinidade, ramificação de suas cadeias poliméricas, baixa estabilidade dos monômeros (xilose, galactose, manose, arabinose e raminose), e à presença de grupos acetilas (SJOSTROM e ALÉN, 1999).

As hemiceluloses estão ligadas à celulose através de grande quantidade de ligações de hidrogênio e seus acoplamentos físicos tornam quase impossível separá-las, sem danos à celulose (COLODETTE, 2005).

Os conteúdos de hemiceluloses presentes em madeiras de eucaliptos adaptadas ao Brasil, Eucalyptus grandis e o híbrido Eucalyptus grandis $x$ Eucalyptus urophylla, são relativamente baixos se comparados aos de outras madeiras de fibra curta, como Eucalyptus globulus e Betula pendula. Entretanto, as hemiceluloses dos eucaliptos são basicamente constituídas de xilanas (PASCOAL NETO et al., 2004), que são solúveis em álcali e água (SJÖSTRÖM e ALÉN, 1999) e, portanto, com potencial de remoção por simples extração. As principais variáveis envolvidas na lixiviação com água e álcali, com o propósito de remover xilanas, são temperatura, tempo e pH (SINGH, 1979).

Durante a polpação kraft da madeira de eucalipto, grandes quantidades de xilanas são dissolvidas e eliminadas junto com o licor negro. De acordo com o cálculo teórico apresentado por Colodette (2005), para a madeira de Eucalyptus grandis, a perda de hemiceluloses durante a polpação kraft pode alcançar $62 \%$ do seu valor original.

A auto-hidrólise dos cavacos acontece pelo fato de a madeira possuir grupos acetilas, que são os constituintes mais facilmente extraíveis, e em meio aquoso esses grupos tendem a diminuir o pH para níveis ácidos. Assim, uma remoção parcial de hemiceluloses pode ser conseguida apenas adicionando água e controlando o tempo e a temperatura dos tratamentos, sem a necessidade de ácidos ou bases mais fortes (COLODETTE, 2005).

Tschirner et al. (2006), estudando a remoção de xilanas, constataram que em condições ácidas $\left(\mathrm{H}_{2} \mathrm{SO}_{4} \quad 0,01-1,0 \% \mathrm{p} / \mathrm{v}\right)$ todas as xilanas foram hidrolisadas; que em condições de autohidrólise (relação água/madeira de $4: 1 \mathrm{~m}^{3} / \mathrm{t}$ ) a hidrólise das xilanas começa a partir de $150^{\circ} \mathrm{C}$; e que em condições alcalinas ( $\mathrm{NaOH} 4 \% \mathrm{w} / \mathrm{w})$ ocorre baixa remoção de xilanas até $170^{\circ} \mathrm{C}$, devendo ser ressaltado que a fração removida se apresenta na forma polimérica (Figura 1).

Em continuidade aos seus estudos, Tschirner et al. (2006) concluíram que o pré-tratamento com auto-hidrólise foi o mais viável para anteceder aos cozimentos, devido à possibilidade de diminuir o tempo dos cozimentos ou reduzir o consumo de reagentes.

Nos estudos que envolvem pré-tratamentos com água, foi constatado que as características físicas e químicas dos materiais tratados influenciam significativamente a remoção dos seus constituintes químicos (celulose, hemiceluloses e lignina). Portanto, são pertinentes os estudos que visam à otimização das principais variáveis dos tratamentos (tempo, temperatura, relação líquido/madeira, concentração de reagentes químicos, aditivos etc.) para a madeira do híbrido de Eucalyptus grandis $x$ Eucalyptus urophylla, uma das mais importantes

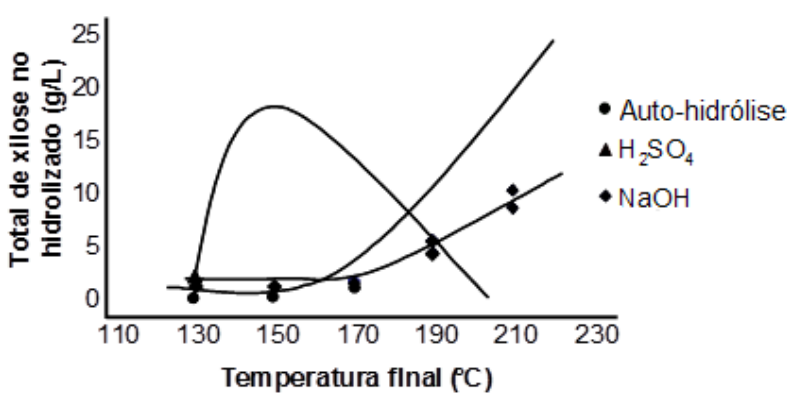

FIGURA 1: Remoção de xilanas da madeira de aspen utilizando os tratamentos: autohidrólise, hidrólise ácida e lixiviação alcalina (TSCHIRNER et al., 2006).

FIGURE 1: Xylans removal from aspen wood using the treatments: self-hydrolysis, acid- hydrolysis and alkaline leaching (TSCHIRNER et al.,2006). 
para a indústria de base florestal brasileira.

O principal objetivo deste estudo foi avaliar o impacto da remoção de hemiceluloses da madeira de eucalipto, por tratamentos de autohidrólise $\left(\mathrm{H}_{2} \mathrm{O}\right)$ dos cavacos, no comportamento do processo kraft subsequente e na branqueabilidade e qualidade da polpa. Foram também determinadas as condições ótimas de tempo e temperatura para extração parcial de hemiceluloses de cavacos de eucalipto.

\section{MATERIAL E MÉTODO}

Foram usados cavacos de madeira de híbridos de Eucalyptus grandis x Eucalyptus urophylla com 7 anos de idade de florestas localizadas no Mato Grosso do Sul. Os cavacos foram classificados em peneiras com malhas de $32 \times 32 \mathrm{~mm}$ e $16 \times 16 \mathrm{~mm}$. Após secos ao ar, foram armazenados em sacos de polietileno, para uniformização e conservação do teor de umidade. Para os estudos de cozimento, os cavacos foram misturados e parte foi moída em moinho tipo Willey. A serragem foi então classificada em peneiras 40/60 mesh, seca ao ar e armazenada em potes de vidro para posteriores análises.

O fluxograma a seguir (Figura 2) mostra o resumo esquemático dos métodos aplicados a esse estudo.

\section{Auto-hidrólise dos cavacos}

O tratamento de auto-hidrólise foi realizado com $200 \mathrm{~g}$ de cavacos, em um digestor batch rotatório, aquecido eletricamente e o tempo e a temperatura de reação foram eletronicamente controlados como mostrado na Tabela 1. Ao final

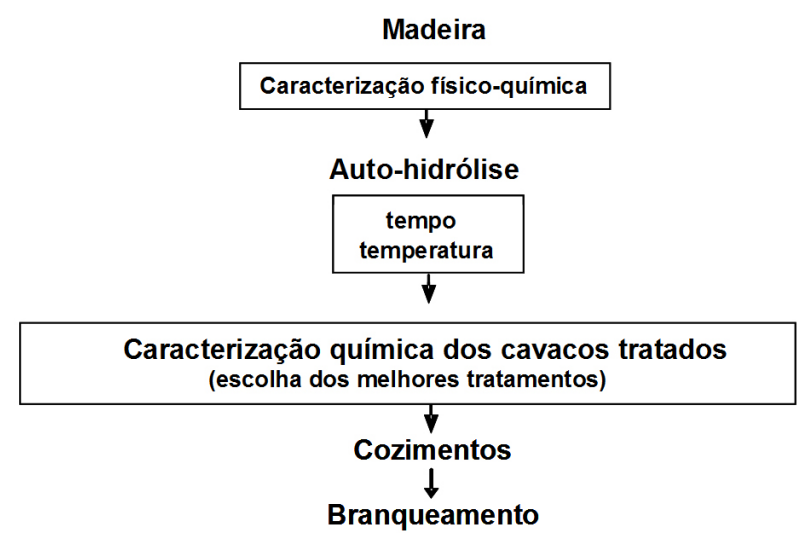

FIGURA 2: Esquema experimental do estudo. FIGURE 2: Experimental model of this study. dos tratamentos, foram coletados os licores residuais para análises e os cavacos foram imediatamente processados pelo cozimento kraft.

\section{Cozimento kraft dos cavacos normais e auto- hidrolisados}

Três condições da auto-hidrólise $(5,15$ e 30 minutos à temperatura), correspondentes aos tratamentos de auto-hidrólise a $170^{\circ} \mathrm{C}$, foram selecionados para seguir com o cozimento kraft. Imediatamente após o estágio de auto-hidrólise, assim que extraído o licor ácido, foi adicionado o licor de cozimento kraft ao digestor. O volume de licor adicionado foi calculado de forma a obter relação licor/madeira de $4: 1 \mathrm{~m}^{3} / \mathrm{t}$, considerando a umidade contida nos cavacos. A carga de álcali efetivo aplicada ao cozimento foi determinada experimentalmente, a fim de obter polpa marrom com número kappa entre 17 e 18.

\section{Branqueamento das polpas derivadas de cavacos normais e de auto-hidrólise}

O branqueamento das polpas produzidas dos cavacos-referência e auto-hidrólise foi realizado com a sequência $\mathrm{O} / \mathrm{OD}(\mathrm{EPO}) \mathrm{DD}$, onde $\mathrm{O} / \mathrm{O}$ (duplo estágio com oxigênio), D (estágio de dioxidação) e EPO (estágio de extração oxidativa com peróxido de hidrogênio), e o resumo das condições do branqueamento estão na Tabela 2.

Um resumo dos métodos e referências usados nesse estudo está descrito na Tabela 3.

TABELA 1: Condições utilizadas no tratamento de auto-hidrólise da madeira.

TABLE 1: Conditions used in the self-hydrolysis treatment of wood.

\begin{tabular}{|c|c|c|c|}
\hline \multicolumn{4}{|c|}{ Condições de auto-hidrólise } \\
\hline $\begin{array}{l}\text { Massa de } \\
\text { cavacos }\end{array}$ & \multicolumn{3}{|c|}{$250 \mathrm{~g}$} \\
\hline $\begin{array}{l}\text { Licor de } \\
\text { cozimento }\end{array}$ & \multicolumn{3}{|c|}{ Somente água } \\
\hline Licor/madeira & \multicolumn{3}{|c|}{$4: 1 \mathrm{~m}^{3} / \mathrm{t}$} \\
\hline Temperatura & $152^{\circ} \mathrm{C}$ & $160^{\circ} \mathrm{C}$ & $170^{\circ} \mathrm{C}$ \\
\hline $\begin{array}{l}\text { Tempo à } \\
\text { temperatura }\end{array}$ & \begin{tabular}{|c|}
$30,45 \mathrm{e}$ \\
$60 \mathrm{~min}$ \\
\end{tabular} & $\begin{array}{l}15,30 \mathrm{e} \\
45 \mathrm{~min}\end{array}$ & $\begin{array}{c}5,10,15, \\
20 \text { e } 30 \mathrm{~min}\end{array}$ \\
\hline $\begin{array}{l}\text { Tempo até } \\
\text { temperatura }\end{array}$ & \multicolumn{3}{|c|}{$90 \mathrm{~min}$} \\
\hline
\end{tabular}


TABELA 2: Condições gerais do branqueamento de polpas oriundas de cavacos auto-hidrolisados.

TABLE 2: General bleaching conditions of pulp from self-hydrolysis chips.

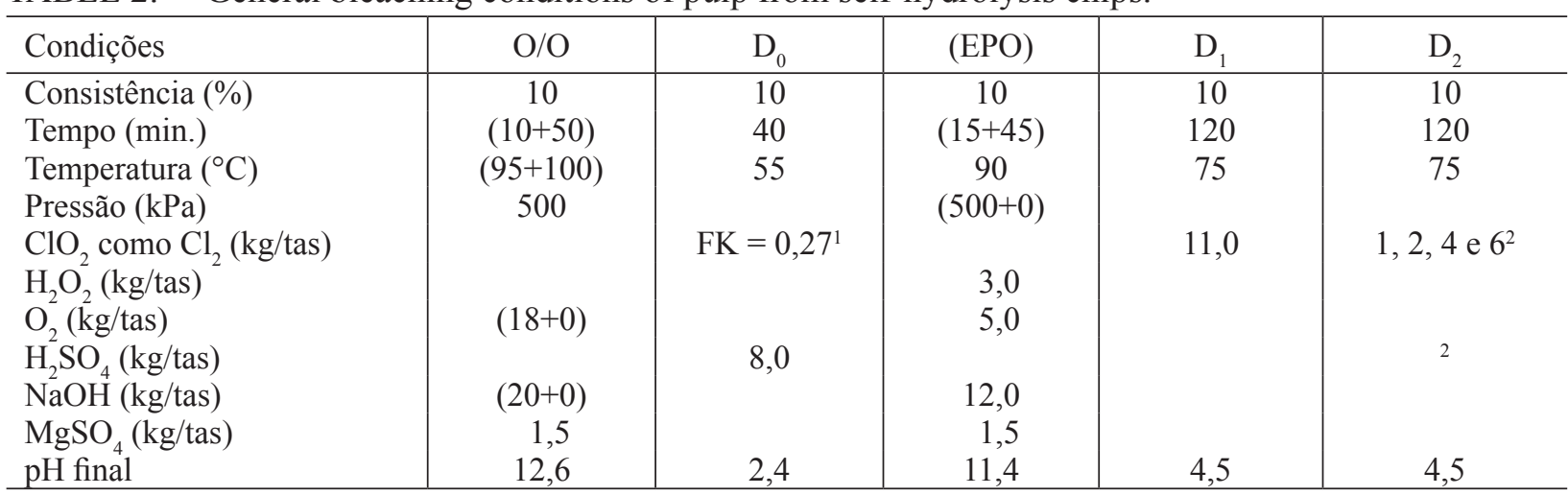

Em que: ${ }^{1} \mathrm{FK}$ = fator kappa; ${ }^{2}$ dosagem para atingir alvura final 92\% ISO; O/O = duplo estágio com oxigênio; D = estágio de dioxidação; EPO = estágio de extração oxidativa com peróxido de hidrogênio.

TABELA 3: Procedimentos analíticos para caracterização físico-química da madeira, licores e polpa celulósica.

TABLE 3: Analytical proceedings for physical and chemical characterization of wood, liquors and cellulosic pulp.

\begin{tabular}{|c|c|}
\hline Parâmetros & Referências \\
\hline \multicolumn{2}{|r|}{ Madeira } \\
\hline Ácidos urônicos & Sundberg et al. (1996) \\
\hline Classificação dos cavacos & SCAN 40:94 \\
\hline Composição de açúcares & HPLC - Método, depois da hidrólise ácida de acordo com Tappi T 249 \\
\hline Densidade aparente & Propriedade LCP \\
\hline Densidade básica & Propriedade LCP \\
\hline Extrativos totais & Tappi T $264 \mathrm{~cm}-97$ \\
\hline Extrativos em etanol/tolueno $(1: 2)$ & Tappi T204 cm-97 \\
\hline Grupos acetilas & Solar et al. (1987) \\
\hline Relação lignina siringila guaiacila & Nitrobenzene oxidation according to Lin e Dence, 1992. \\
\hline \multicolumn{2}{|r|}{ Polpa } \\
\hline Ácidos hexenurônicos (HeXas) & Vuorinen et al. (1996) \\
\hline Alvura & Tappi T525 om-92 \\
\hline Composição de açúcares & HPLC - Método, depois da hidrólise, ácida de acordo com Tappi T 249 \\
\hline Formação manual de folhas & Tappi T 218 sp-97 \\
\hline Número Kappa & Tappi T $236 \mathrm{~cm}-85$ \\
\hline Pentosanas & Tappi T223 cm-84 \\
\hline Rejeitos da polpação & Gravimétrico - Propriedade LCP \\
\hline Rendimento da polpação & Gravimétrico - Propriedade LCP \\
\hline Reversão de alvura & Tappi UM 200 \\
\hline Viscosidade & Tappi T230 om-94 \\
\hline \multicolumn{2}{|r|}{ Licor Negro } \\
\hline Conteúdo de sódio & Tappi T25 cm-85 \\
\hline Conteúdo de sulfeto & Tappi $625 \mathrm{~cm}-85$ \\
\hline Residual de álcali & Tappi T625 cm-85 \\
\hline \multicolumn{2}{|r|}{ Efluente } \\
\hline $\begin{array}{l}\text { Análises de soluções e residuais de dióxido } \\
\text { de cloro e peróxido de hidrogênio }\end{array}$ & McDonald (1967) \\
\hline $\mathrm{AOX}$ & Scan -P 69:94 \\
\hline Carbono orgânico total & APHA - Standard Methods, 1995. \\
\hline Cor & CPPA H.5 \\
\hline $\mathrm{DQO}$ & CPPA H.3 \\
\hline $\mathrm{DBO}$ & CPPA H.1 \\
\hline
\end{tabular}




\section{RESULTADOS E DISCUSSÃO}

Os resultados médios da caracterização físico-química da madeira, provenientes de duas repetições, estão mostrados na Tabela 4.

Os valores de densidade básica e aparente são típicos de madeiras de eucalipto utilizadas para fabricação de celulose kraft no Brasil. Os dados das análises químicas da madeira indicam se tratar de material com baixo teor de extrativos $(\sim 1,5 \%)$, uma vez que para os clones industriais de eucaliptos brasileiros, de modo geral, esse valor varia entre 1 e 4\% (GOMIDE et al., 2005). O teor total de hemiceluloses da madeira em estudo foi de $21,4 \%$, resultado este, similar, aos observados por Pascoal Neto et al. (2004), para outras madeiras de Eucalyptus grandis e híbridos de Eucalyptus grandis x Eucalyptus urophylla. São entendidas como hemiceluloses o somatório das xilanas + galactanas + mananas + arabinanas + ácidos urônicos + grupos acetilas + pequena parte de glicanas. O conteúdo de ácidos urônicos de 4,3\% está dentro da faixa relatada em outros estudos, porém o teor de grupos acetilas $(2,1 \%)$ se mostrou ligeiramente baixo (PASCOAL NETO et al., 2004). O material apresentou teor de lignina normal, em torno de $27,8 \%$, sendo a variabilidade reportada em outros estudos (GOMIDE et al., 2005) de 27,5 $31,7 \%$. A relação lignina siringila/guaiacila de 2,07 pode ser considerada baixa, pois estudos com outras madeiras de eucalipto revelam valores na faixa de

\section{2,1-4,7 (MOKFIENSKI et al., 2003).}

\section{Auto-hidrólise dos cavacos}

O aumento do tempo da auto-hidrólise de 5 para 30 minutos à temperatura de $170^{\circ} \mathrm{C}$ aumentou a remoção de hemiceluloses (medidas como pentosanas) dos cavacos. O conteúdo de pentosanas diminuiu de 14,2 para $6,3 \%$ e, consequentemente, diminuiu o rendimento do processo (Tabela 5).

Esses resultados demonstram que as pentosanas podem facilmente ser removidas dos cavacos de madeira de eucalipto por um estágio de auto-hidrólise e que a eficiência da remoção é proporcional à intensidade (tempo) do tratamento. Considerando-se que a madeira original possuía $15,5 \%$ de pentosanas (Tabela 4), o tratamento de auto-hidrólise por 30 minutos removeu cerca de $60 \%$ das pentosanas originais da madeira.

A água usada na auto-hidrólise transformouse em licor ácido durante o tratamento e apresentou pH na faixa de 2,9-3,2 no final da reação, dependendo da condição empregada. $\mathrm{O}$ pH baixo foi causado pela hidrólise ácida dos grupos acetilas, os quais estão ligados à estrutura química das xilanas.

A madeira utilizada neste estudo apresentou conteúdo original de lignina de 27,75 (Tabela 4) e o tratamento auto-hidrólise causou pequeno aumento desse teor, o que pode ser explicado pela remoção parcial das pentosanas, com consequente diminuição da base seca e aumento do teor proporcional de lignina.

TABELA 4: Resultados das análises físico-químicas da madeira.

TABLE 4: Results of physical and chemical analysis of wood.

\begin{tabular}{|c|c|c|}
\hline \multicolumn{2}{|l|}{ Características } & Média \\
\hline \multicolumn{2}{|l|}{ Densidade básica $\left(\mathrm{kg} / \mathrm{m}^{3}\right)$} & 528 \\
\hline \multicolumn{2}{|l|}{ Densidade aparente dos cavacos $\left(\mathrm{kg} / \mathrm{m}^{3}\right)$} & 193 \\
\hline \multicolumn{2}{|l|}{ Celulose $(\%)^{1}$} & 49,38 \\
\hline \multirow{7}{*}{ Hemiceluloses $(\%)$} & Xilanas & 12,03 \\
\hline & Galactanas & 0,97 \\
\hline & Mananas & 0,92 \\
\hline & Arabinanas & 0,14 \\
\hline & Grupos Acetila & 2,07 \\
\hline & Ácidos Urônicos & 4,34 \\
\hline & Total $^{2}$ & 21,39 \\
\hline \multicolumn{2}{|l|}{ Extrativos (\% em etanol/tolueno, 1:2) } & 1,50 \\
\hline \multicolumn{2}{|l|}{ Pentosanas $(\%)$} & 15,50 \\
\hline \multicolumn{2}{|l|}{ Lignina $(\%)$} & 27,75 \\
\hline \multirow{3}{*}{ Relação S:G } & Siringila, gmol & 2,52 \\
\hline & Guaiacila, gmol & 1,22 \\
\hline & S:G & 2,07 \\
\hline
\end{tabular}

Em que: ${ }^{1}$ Celulose $(\%)=$ glicanas totais (\%) - mananas (\%); ${ }^{2}$ inclui as glicanas que constituem as hemiceluloses. 
TABELA 5: Resultados dos tratamentos de auto-hidrólise a $170^{\circ} \mathrm{C}$ por $5-30$ minutos.

TABLE 5: Results of self-hydrolysis treatments at the $170^{\circ} \mathrm{C}$ from 5 to 30 minutes.

\begin{tabular}{|c|c|c|c|c|c|c|}
\hline \multirow{2}{*}{\multicolumn{2}{|c|}{ Parâmetros }} & \multicolumn{5}{|c|}{ Tempos de Tratamento a $170^{\circ} \mathrm{C}$, min } \\
\hline & & 5 & 10 & 15 & 20 & 30 \\
\hline \multicolumn{2}{|c|}{ Rendimento total (\%) } & 94,7 & 92,4 & 88,9 & 87,2 & 83,5 \\
\hline \multicolumn{2}{|c|}{ Teor de pentosanas remanescentes $(\%)$} & 14,2 & 12,6 & 10,3 & 8,5 & 6,3 \\
\hline \multicolumn{2}{|c|}{$\mathrm{pH}$ licor residual } & 3,2 & 3,1 & 2,8 & 2,9 & 2,9 \\
\hline \multicolumn{2}{|c|}{ Sólidos no licor (\%) } & 1,3 & 1,8 & 2,4 & 2,7 & 3,3 \\
\hline \multirow{3}{*}{ Lignina (\%) } & Insolúvel & 24,5 & 24,2 & 25,4 & 26,7 & 26,8 \\
\hline & Solúvel & 2,6 & 2,7 & 2,2 & 2,3 & 1,9 \\
\hline & Total & 27,1 & 26,9 & 27,6 & 29,0 & 28,7 \\
\hline
\end{tabular}

Também foram realizados estágios de auto-hidrólise a temperaturas mais baixas, 152 e $160^{\circ} \mathrm{C}$, para verificar o comportamento das pentosanas (Tabela 6). Verifica-se menor remoção de pentosanas da madeira nos tratamentos a $152^{\circ} \mathrm{C}$ e $160^{\circ} \mathrm{C}$, mesmo em tempos mais longos de reação. As máximas remoções de pentosanas foram 6,4 e $27,1 \%$ a 152 e $160^{\circ} \mathrm{C}$, respectivamente, enquanto a $170^{\circ} \mathrm{C}$ a remoção foi próxima de $60 \%$. $\mathrm{O} \mathrm{pH}$ final dos tratamentos a 152 e $160^{\circ} \mathrm{C}(3,1 \mathrm{e}$ 3,2 , respectivamente) foi apenas ligeiramente maior que os valores de $\mathrm{pH}$ dos tratamentos a $170^{\circ} \mathrm{C}$ (2,9-3,2). Portanto, concluiu-se que o efeito da temperatura é decisivo na remoção das pentosanas.

Com o aumento do tempo e da temperatura de reação ocorreu maior remoção de pentosanas e, paralelamente, ocorreu queda de rendimento. $\mathrm{Na}$ Tabela 6 estão os resultados de remoção de pentosanas na madeira e o rendimento final dos tratamentos, respectivamente. Tendo em vista a baixa remoção de pentosanas dos cavacos nas temperaturas de 152 e $160^{\circ} \mathrm{C}$, optou-se por utilizar os cavacos auto-hidrolisados a $170^{\circ} \mathrm{C}$ nos estudos de cozimento (Tabela 5). A demanda de álcali efetivo para cozinhar os cavacos auto-hidrolisados até número kappa 17-18 variou de 14,4 a 15,1\% como $\mathrm{NaOH}$, sendo o valor mais baixo para as polpas auto-hidrolisadas pelo tempo de 30 minutos (Tabela
7). Portanto a auto-hidrólise da madeira não teve efeito na demanda de álcali durante o cozimento kraft para esse grau de deslignificação.

Observou-se que o rendimento dos cozimentos das madeiras auto-hidrolisadas variou de 44,9 a $45,3 \%$, sendo o valor mais baixo de rendimento correspondente ao tempo mais longo de auto-hidrólise (30 minutos). O rendimento do cozimento-referência foi de 50,9\%. Portanto, verificou-se que a remoção das hemiceluloses da madeira diminuiu o rendimento da polpação kraft subsequente. Vale a pena notar que os rendimentos totais, incluindo o rendimento da auto-hidrólise mais o rendimento do cozimento, foram ainda mais baixos (37,5 - 42,9\%). Pode-se inferir que as pentosanas remanescentes nos cavacos após a autohidrólise são muito mais sensíveis à polpação kraft que aquelas originais da madeira.

Ao final do cozimento, os conteúdos de lignina das polpas derivadas de cavacos autohidrolisados foram mais baixos $(0,79-0,95 \%)$ do que poderia se esperar para polpas de kappa 17-18 e muito menores que o conteúdo de lignina da polpareferência $(1,9 \%)$.

Algumas mudanças significativas foram observadas nas características do licor negro derivado do cozimento kraft de cavacos autohidrolisados, em relação à referência (Tabela 4). $\mathrm{O}$ teor de sólidos do licor negro kraft aumentou

TABELA 6: Resultados dos tratamentos de auto-hidrólise a $152^{\circ} \mathrm{C}$ e $160^{\circ} \mathrm{C}$ por $15-60$ minutos.

TABLE 6: Results of self-hydrolysis treatments at the $152^{\circ} \mathrm{C}$ and $160^{\circ} \mathrm{C}$ from 15 to 60 minutes.

\begin{tabular}{ccccc}
\hline $\begin{array}{c}\text { Temperatura } \\
\left({ }^{\circ} \mathrm{C}\right)\end{array}$ & $\begin{array}{c}\text { Tempo a } \\
\text { Temperatura (min.) }\end{array}$ & $\begin{array}{c}\text { Rendimento. Total } \\
(\%)\end{array}$ & $\begin{array}{c}\text { Pentosanas } \\
(\%)\end{array}$ & pH Licor Residual \\
\hline 152 & 30 & 96,6 & 15,6 & 3,5 \\
152 & 45 & 94,4 & 14,7 & 3,4 \\
152 & 60 & 93,1 & 14,5 & 3,1 \\
160 & 15 & 96,3 & 15,8 & 3,6 \\
160 & 30 & 91,5 & 13,0 & 3,3 \\
160 & 45 & 87,1 & 11,3 & 3,2 \\
\hline
\end{tabular}

Ci. Fl., v. 21, n. 3, jul.-set., 2011 
TABELA 7: Resultados do cozimento kraft de cavacos-referência e auto-hidrolisados - características da polpa.

TABLE 7: Kraft cooking results of reference chips and self-hydrolysis - pulp characteristics.

\begin{tabular}{|c|c|c|c|c|c|}
\hline \multirow{2}{*}{\multicolumn{2}{|c|}{ Parâmetros }} & \multicolumn{4}{|c|}{ Tempo de Auto-hidrólise (min.) } \\
\hline & & 0 & 5 & 15 & 30 \\
\hline \multicolumn{2}{|c|}{ Álcali efetivo $(\% \mathrm{NaOH})$} & 14,8 & 15,1 & 14,8 & 14,4 \\
\hline \multicolumn{2}{|l|}{ Número kappa } & 18 & 17 & 17 & 17 \\
\hline \multirow{3}{*}{$\begin{array}{l}\text { Rendimento do } \\
\text { Cozimento (\%) }\end{array}$} & Depurado & 50,6 & 45,2 & 45,5 & 44,9 \\
\hline & Rejeitos & 0,3 & 0,1 & 0,1 & 0,0 \\
\hline & Total & 50,9 & 45,3 & 45,6 & 44,9 \\
\hline \multicolumn{2}{|c|}{ Rendimento de auto-hidrólise + polpação (\%) } & 50,9 & 42,9 & 40,5 & 37,5 \\
\hline \multicolumn{2}{|l|}{ Viscosidade (cP) } & 58 & 69 & 61 & 48 \\
\hline \multicolumn{2}{|l|}{ Pentosanas (\%) } & 17 & 7,3 & 3,3 & 2,0 \\
\hline \multicolumn{2}{|c|}{ Ácidos hexenurônicos (mmol $/ \mathrm{kg})$} & 0,92 & 0,22 & 0,12 & 0,07 \\
\hline \multirow{3}{*}{ Lignina (\%) } & Insolúvel & 1,3 & 0,3 & 0,6 & 0,5 \\
\hline & Solúvel & 0,6 & 0,5 & 0,4 & 0,3 \\
\hline & Total & 1,9 & 0,9 & 1,0 & 0,8 \\
\hline \multirow{5}{*}{$\begin{array}{l}\text { Licor negro } \\
\text { residual }\end{array}$} & Sólidos totais (\%) & 14,8 & 15,3 & 15,3 & 15,5 \\
\hline & Sólidos orgânicos (\%) & 60,8 & 61,7 & 62,2 & 62,4 \\
\hline & Sólidos inorgânicos (\%) & 39,2 & 38,3 & 37,9 & 37,6 \\
\hline & Álcali efetivo residual (g/L) & 0 & 0 & 0 & 0 \\
\hline & Poder calorífico (kcal/kg sólidos) & 3.464 & 3.631 & 3.813 & 3.887 \\
\hline
\end{tabular}

de 14,8 (referência) para 15,3-15,5\% (cavacos auto-hidrolisados). Quanto maior o tempo de autohidrólise, maior o teor de sólidos no licor negro, refletindo o menor rendimento da polpação kraft. A relação orgânicos/inorgânicos do licor negro tendeu a aumentar ligeiramente com o tempo crescente da auto-hidrólise. O poder calorífico do licor negro aumentou em $12,2 \%$ para os cavacos auto-hidrolisados por 30 minutos, em relação aos cavacos-referência. Este resultado se justifica, dado que as hemiceluloses apresentam apenas a metade do poder calorífico da lignina.

\section{Deslignificação com oxigênio das polpas oriundas de cavacos auto-hidrólise}

A redução do número kappa durante a deslignificação com oxigênio em duplo estágio $(\mathrm{O} / \mathrm{O})$ foi substancialmente mais elevada para as polpas produzidas com cavacos auto-hidrolisados (Tabela 8 ). Este fato ocorreu porque as polpas tinham teores muito mais baixos de ácidos hexenurônicos, HexAs $(5,1-20,1 \mathrm{mmol} / \mathrm{kg})$ que a referência $(52,4 \mathrm{mmol} /$ $\mathrm{kg}$ ). Os HexAs não reagem com o oxigênio e não contribuem para redução do número kappa durante a deslignificação com oxigênio (VUORINEN et al., 1996).

As viscosidades das polpas, medidas após deslignificação com oxigênio, foram mais baixas para as das polpas produzidas dos cavacos auto- hidrolisados. A viscosidade tendeu a diminuir com o aumento do tempo de auto-hidrólise e a seletividade e o ganho de alvura no estágio $\mathrm{O} / \mathrm{O}$ aumentaram, fato este que pode ser explicado pela elevada eficiência de deslignificação dessa etapa com as polpas derivadas dos cavacos auto-hidrolisados.

A perda de rendimento na deslignificação com oxigênio não foi muito influenciada pelo tempo de auto-hidrólise, variando de 1,8 a 2,2\%. Este resultado indicou que a deslignificação com oxigênio foi muito seletiva para a fração de lignina da polpa. De fato, a perda de rendimento por unidade de kappa removido durante o estágio $\mathrm{O} / \mathrm{O}$ foi muito mais baixa para as polpas derivadas dos cavacos auto-hidrolisados por 30 minutos, o que pode ser explicado pelo menor teor de pentosanas presentes nessas polpas, em relação à referência.

\section{Branqueamento das polpas oriundas de cavacos auto-hidrólise}

Para realizar uma comparação lógica, todas as sequências de branqueamento foram realizadas em condições operacionalmente similares, mantendo constante o fator kappa de 0,27 no primeiro estágio de branqueamento e variando a dosagem de dióxido de cloro no segundo e terceiro estágios, a fim de conseguir alvura final de 92\% ISO. Nenhum tipo de otimização foi feita nesta primeira fase do estudo. 
TABELA 8: Resultados da deslignificação com oxigênio de polpas oriundas de cavacos-referência e autohidrolisados.

TABLE 8: Oxygen delignification results of pulp from reference and self-hydrolysis chips.

\begin{tabular}{|c|c|c|c|c|}
\hline \multirow{2}{*}{ Parâmetros } & \multicolumn{4}{|c|}{ Tempo de Auto-hidrólise, min } \\
\hline & 0 & 5 & 15 & 30 \\
\hline $\mathrm{NaOH}(\%)$ & 2,0 & 2,0 & 2,0 & 2,0 \\
\hline Kappa inicial & 18,1 & 16,8 & 17,4 & 16,8 \\
\hline Kappa final & 10,2 & 5,9 & 5,8 & 4,2 \\
\hline HexAs inicial (mmol/kg) & 52,4 & 20,1 & 9,1 & 5,1 \\
\hline HexAs final $(\mathrm{mmol} / \mathrm{kg})$ & 49,3 & 18,5 & 8,5 & 4,9 \\
\hline Viscosidade inicial (cP) & 58,4 & 69,4 & 61,1 & 48,0 \\
\hline Viscosidade final (cP) & 35,7 & 33,2 & 30,4 & 22,4 \\
\hline Alvura inicial (\%ISO) & 30,1 & 27,4 & 26,0 & 26,2 \\
\hline Alvura final (\%ISO) & 45,7 & 50,5 & 47,1 & 54,5 \\
\hline Redução do kappa (\%) & 43,6 & 64,9 & 66,7 & 75,0 \\
\hline Remoção de HexAs (\%) & 5,9 & 8,0 & 6,6 & 5,8 \\
\hline Redução de viscosidade (\%) & 38,9 & 52,2 & 50,2 & 53,3 \\
\hline Ganho de alvura (\%) & 15,6 & 23,1 & 21,1 & 28,3 \\
\hline Seletividade $=\Delta$ visc $/ \Delta$ kappa & 2,9 & 3,3 & 2,7 & 2,0 \\
\hline Rendimento (\%) & 97,9 & 97,8 & 98,1 & 98,2 \\
\hline
\end{tabular}

Os custos de branqueamento variaram de US\$27.46/tas (tonelada de celulose absolutamente seca) para a polpa-referência, a US\$20.97/tas para a polpa derivada dos cavacos auto-hidrolisados durante o tempo de 30 minutos (Tabela 9). Quanto maior o tempo de auto-hidrólise menor o custo do branqueamento. $\mathrm{O}$ menor custo de reagentes químicos para branqueamento das polpas autohidrolisadas em relação à referência derivou do menor número kappa da polpa que entrou na planta de branqueamento. Esse menor número kappa foi devido ao fato de a deslignificação com oxigênio ter sido mais eficiente para as polpas com quantidades muito baixas de HexAs.

As polpas produzidas dos cavacos autohidrolisados mostraram menor branqueabilidade que a polpa-referência, ou seja, elas apresentaram maior demanda de cloro ativo por unidade de kappa removido durante o branqueamento que a referência. Isto era esperado, visto que as polpas que entram no branqueamento com números kappa mais baixos têm menor disponibilidade de grupos hidroxílicos fenólicos livres para reagirem com o dióxido de cloro. Neste caso particular, a polpareferência entrou na planta de branqueamento com um número kappa de 10,2, valor este quase duas vezes maior que o kappa das polpas derivadas de cavacos auto-hidrolisados $(4,2-5,9)$.

A reversão de alvura variou de 1,3 a 1,7\%, sendo os valores mais baixos encontrados para a polpa derivada dos cavacos auto-hidrolisados por 30 minutos. Em geral, as polpas derivadas de cavacos auto-hidrolisados apresentaram menor reversão de alvura, resultado que pode ser explicado pelos mais baixos valores de HexAs presentes nessas polpas. Em geral, os valores da reversão de alvura encontrados são plenamente aceitáveis para polpas de eucalipto de mercado, devendo ser ressaltado que as diferenças observadas entre as várias amostras estão, provavelmente, dentro do erro experimental. $\mathrm{O}$ próprio teste de medição de alvura está sujeito a erro de $0,5 \%$ ISO devido ao método. Também, os valores das coordenadas $\mathrm{L}^{*} \mathrm{a} \mathrm{b}^{*}$ não variaram entre as diferentes polpas.

As viscosidades finais das polpas oriundas dos cavacos auto-hidrolisados foram mais baixas que aquela da polpa-referência. A polpa derivada dos cavacos auto-hidrolisados por 30 minutos apresentou viscosidade final abaixo do mínimo requerido para fabricação de determinadas classes de papel $(11,2 \mathrm{cP})$. Entretanto, as polpas auto-hidrolisadas por 5 e 15 minutos resultaram em viscosidades razoáveis $(16,3$ e 16,4 cP). Os rendimentos totais do branqueamento, incluindo o rendimento do estágio $\mathrm{O} / \mathrm{O}$, variaram na escala de 95,2 a $95,7 \%$, sendo o valor mais baixo para cavacos-referência e o valor mais elevado para os cavacos auto-hidrolisados por 30 minutos.

As cargas dos efluentes de branqueamento também estão na Tabela 9. Observa-se que as cargas deAOX, DQO e a cor dos efluentes de branqueamento derivados dos cavacos auto-hidrolisados foram mais 
TABELA 9: Resultados do branqueamento O/OD(EPO)DD de polpas oriundas de cavacos-referência e auto-hidrolisados.

TABLE 9: O/OD(EPO)DD pulp bleaching results from reference chips and self-hydrolysis chips.

\begin{tabular}{|c|c|c|c|c|}
\hline \multirow{2}{*}{ Resultados } & \multicolumn{4}{|c|}{ Tempo de Auto-hidrólise (min.) } \\
\hline & 0 & 5 & 15 & 30 \\
\hline $\mathrm{ClO}_{2}\left(\%\right.$ como $\left.\mathrm{Cl}_{2}\right)$ & 3,98 & 2,79 & 2,77 & 2,33 \\
\hline $\mathrm{H}_{2} \mathrm{O}_{2}(\%)$ & 0,30 & 0,30 & 0,30 & 0,30 \\
\hline $\mathrm{O}_{2}(\%)^{1}$ & 2,30 & 2,30 & 2,30 & 2,30 \\
\hline $\mathrm{NaOH}(\%)$ & 1,20 & 1,20 & 1,20 & 1,20 \\
\hline $\mathrm{H}_{2} \mathrm{SO}_{4}(\%)$ & 0,83 & 0,65 & 0,55 & 0,55 \\
\hline $\mathrm{MgSO}_{4}(\%)^{1}$ & 0,30 & 0,30 & 0,30 & 0,30 \\
\hline $\mathrm{ClO}_{2}\left(\%\right.$ como $\left.\mathrm{ClO}_{2}\right)$ & 1,51 & 1,06 & 1,05 & 0,89 \\
\hline Cloro ativo total $(\%)^{2}$ & 4,61 & 3,42 & 3,39 & 2,96 \\
\hline Branqueabilidade, $\Delta \mathrm{K} / \%$ Cloro ativo & 2,21 & 1,73 & 1,71 & 1,42 \\
\hline Custo de reagentes (US\$/t a.s.) ${ }^{3}$ & 27,46 & 22,80 & 22,62 & 20,97 \\
\hline Alvura (\% ISO) & 92,10 & 92,00 & 92,00 & 92,10 \\
\hline Reversão de alvura (\% ISO) & 90,40 & 90,50 & 90,50 & 90,80 \\
\hline Reversão (\% ISO) & 1,70 & 1,50 & 1,50 & 1,30 \\
\hline $\mathrm{L} *(\%)$ & 98,10 & 98,20 & 98,30 & 98,30 \\
\hline$a^{*}(\%)$ & $-0,10$ & $-0,04$ & 0,06 & 0,16 \\
\hline$b^{*}(\%)$ & 2,40 & 2,60 & 2,70 & 2,50 \\
\hline Viscosidade da polpa (mPas) & 26,30 & 16,40 & 16,30 & 11,20 \\
\hline Rendimento do branqueamento $(\%)^{4}$ & 95,20 & 95,30 & 95,60 & 95,70 \\
\hline Número kappa - Estágio O/O & 10,20 & 5,90 & 5,80 & 4,20 \\
\hline DQO efluente combinado $\left(\mathrm{kg} \mathrm{O}_{2} /\right.$ tas $)$ & 18,20 & 16,10 & 15,00 & 11,00 \\
\hline Cor do efluente combinado ( $\mathrm{kg} \mathrm{Pt} / \mathrm{tas})$ & 9,40 & 9,00 & 8,80 & 7,40 \\
\hline AOX efluente combinado ( $\mathrm{g} \mathrm{Cl}^{-} /$tas) & 0,33 & 0,31 & 0,29 & 0,16 \\
\hline
\end{tabular}

Em que: ${ }^{1}$ Inclusão de oxigênio e magnésio aplicados no estágio $\mathrm{O} / \mathrm{O} ;{ }^{2} \mathrm{Cloro}$ ativo total $=\left(\mathrm{ClO}_{2} * 2,63+\mathrm{H}_{2} \mathrm{O}_{2} * 2,09\right.$ $\left.+\mathrm{O}_{3} * 2,5\right) ;{ }^{3}$ Calculado para alvura de 92,0 \% ISO, exatamente; ${ }^{4}$ Inclui a perda de rendimento depois do estágio O/O, usando equações baseadas na medição de COT - Perda de rendimento O/O(\%)=0,0719*COT (kg C/t a.s. $)+0,9844$ e Perda de rendimento no branqueamento $(\%)=0,0812 * \mathrm{COT}(\mathrm{kg} \mathrm{C} / \mathrm{t}$ a.s. $)+1,913$.

baixas que aqueles da polpa-referência. De fato, o efluente de branqueamento derivado dos cavacos auto-hidrolisados por 30 minutos apresentou valores de DQO, cor e AOX de 39,5, 21,3 e 51,5\% inferiores aos do branqueamento da polpa-referência. Este fato é explicado pelo número kappa mais baixo da polpa derivada dos cavacos auto-hidrolisados. As polpas de cavacos auto-hidrolisados por 5 e 15 minutos também resultaram em efluentes de menor carga que o da polpa-referência.

\section{CONCLUSÕES}

O processo de auto-hidrólise permitiu remoção de até $60 \%$ do teor de pentosanas da madeira com tratamento a $170^{\circ} \mathrm{C}$, por 30 minutos;

As temperaturas de auto-hidrólise de 160 e $152^{\circ} \mathrm{C}$, mesmo com tempos mais prolongados de reação (até 60 minutos), não foram eficazes na remoção de pentosanas;

Os cavacos auto-hidrolisados por 30 minutos 
a $170^{\circ} \mathrm{C}$ resultaram em polpa com aproximadamente $6 \%$ menos rendimento e $88 \%$ menos pentosanas que os cavacos-referência. Se forem considerados os rendimentos da auto-hidrólise e da polpação, os mesmos produziram rendimento total $13,4 \%$ inferior ao de referência;

O licor negro derivado do cozimento kraft de cavacos auto-hidrolisados apresentou maior teor de sólidos totais e de sólidos orgânicos e maior poder calorífico que o licor de cavacos-referência; A eficiência da deslignificação com oxigênio da polpa derivada de cavacos auto-hidrolisados por 30 minutos foi de $75 \%$, contra $43,6 \%$ da polpareferência;

O custo para branquear a polpa de cavacos auto-hidrolisados por $30 \mathrm{~min}$ a $92 \%$ ISO, com a sequência O/OD(EPO)DD, foi US\$ 7/tas mais baixo que o da polpa-referência;

A estabilidade de alvura, as coordenadas de cor $\mathrm{L}^{*} \mathrm{a} * \mathrm{~b} *$ e os valores de rendimento do branqueamento das polpas derivadas de cavacos auto-hidrolisados foram similares ou melhores que os da referência. As viscosidades finais das polpas derivadas de cavacos auto-hidrolisados foram mais baixas, porém aceitáveis;

As cargas poluentes dos efluentes de branqueamento das polpas derivadas de cavacos auto-hidrolisados foram substancialmente mais baixas que as da polpa-referência.

\section{REFERÊNCIAS BIBLIOGRÁFICAS}

APHA. Standard Methods for Examination of Water and Wastewater, 19. Washington: APHA, AWWA, WPCF, 1995.

COLODETTE, J. L. Wood chemistry course. Viçosa: UFV, 2005. 230 p. (apostila).

CPPA - Canadian Pulp and Paper Association. Technical Section Standard Test Methods. Montreal: 1986.

DENCE, C. W.; LIN, S. Y.; TIMELL, T. E. Methods in lignin chemistry. New York: Springer-Verlag, 1992. p. 301-321.

GOMIDE, J. L. et al. Caracterização tecnológica, para produção de celulose, da nova geração de clones de eucalipto do Brasil. Revista Árvore, Viçosa, v. 29, n. 1, p. 129-137, 2005.

LCP - Normas Internas do Laboratório de Celulose e Papel. Viçosa: Laboratório de Celulose e Papel, 2007.

MCDONALD, R. G. Pulp e paper manufacture: kraft pulping. 2. ed. New York: McGraw-hill Book Company, 1967. p. 628-725. v. 1.

MOKFIENSKI, A. et al. Importância da densidade e do teor de carboidratos totais da madeira de eucalipto no desempenho da linha de fibra. In: COLÓQUIO INTERNACIONAL SOBRE CELULOSE KRAFT DE EUCALIPTO, 1., 2003, Viçosa. Anais... UFV: Viçosa, 2003. p. 15-38.

PASCOAL NETO, C. et al. Bulk and surface chemical composition of ECF-bleached hardwood kraft pulp fibres: A comparative study. Nordic Pulp e Paper Research Journal, Stockholm, v. 19, n. 4, p. 513-520, 2004.

ROOKS, A. Technology summit II targets: The challenge of deployment. Solutions, Norcross. v. 87, n. 6. p. 49-50, 2004.

SCAN - Test Methods. Stockholm: Scandinavian Pulp, Paper and Board Testing Committee, 1989.

SINGH, R. P. The bleaching of pulp. 3rd ed. Atlanta: Tappi Press, 1979. 694 p.

SJÖSTRÖM, E.; ALÉN, R. Analytical methods in wood chemistry, pulping and papermaking.15th ed.1999. Berlin: SpringerVerlag, 1999. 316 p.

SOLAR, R.; KACIK, F.; MELCER, I. Simple semimicro method for the determination of o-acetyl groups in wood and related materials. Nordic Pulp and Paper Research Journal, n. 4, p. 139-141, 1987.

SUNDBERG, A. K. et al. Determination of hemicelluloses and pectins in wood and pulp fibers by acid methanolysis and gas chromatography. Nordic Pulp and Paper Research Journal, n. 11, p. 216-220, 1996.

TAPPI - TECHINICAL ASSOCIATION OF THE PULP AND PAPER INDUSTRY. Tappi Standard Methods, Atlanta: TAPPI, 1993, 1996 e 2000.

TSCHIRNER, U.; AL-DAJANI, W. W.; JENSEN, T. Pre-extraction of hemicelluloses and subsequent pulping of Aspen Chips. In: MEMORIAL INTERNATIONAL SYMPOSIUM ON PAST ACHIEVEMENTS E FUTURE CHALLENGES IN PULPING AND BLEACHING, 1., 2006, Vancouver. Proceedings... Vancouver: ESPRA, 2006.

VUORINEN, T. et al. Selective hydrolysis of hexenuronic acid groups and its application in ECF and TCF bleaching of kraft pulps. In: INTERNATIONAL PULP BLEACHING CONFERENCE, 1996, Atlanta. Proceedings... Atlanta: TAPPI PRESS, 1996. p. 43-51. 